\title{
Relações funcionais entre precipitação provável e média em regiões e climas brasileiros
}

\author{
Jorge Luiz Moretti de Souza(1), Daniela Jerszurki(1) e Emanuelly Cristine Damazio(1)
} (1)Universidade Federal do Paraná, Departamento de Solos e Engenharia Rural, Rua dos Funcionários, no 1.540, CEP 80035-050 Curitiba, PR.
E-mail: jmoretti@ufpr.br, djerszurki@ufpr.br, emanuelly.damazio@bol.com.br

Resumo - O objetivo deste trabalho foi determinar os parâmetros estatísticos da função densidade de probabilidade (FDP) com melhor ajuste aos valores decendiais de precipitação pluvial observados em diversas localidades brasileiras, e também determinar a relação entre precipitação provável (75\% de probabilidade, $\mathrm{P}_{75 \%}$ ) e precipitação média $(\overline{\mathrm{P}})$ nestas localidades. Foram avaliadas cinco FDPs (normal, triangular, gama, exponencial e uniforme), ajustadas a dados provenientes de 43 municípios, de oito estados, em quatro regiões brasileiras. As localidades foram avaliadas isoladamente ou agrupadas de acordo com estados ou tipos climáticos. O teste de aderência de Kolmogorov-Smirnov foi utilizado para avaliar o ajuste estatístico das FDPs às séries de dados. As distribuições gama e exponencial foram as que mais frequentemente melhor se ajustaram às séries de precipitação pluvial decendial (41,2 e 30,8\%, respectivamente). As relações funcionais mais promissoras entre $\overline{\mathrm{P}}$ e $\mathrm{P}_{75 \%}$ foram obtidas nos climas Cwa $\left(\mathrm{R}^{2}=0,82\right)$, Aw $\left(\mathrm{R}^{2}=0,70\right)$, As $\left(\mathrm{R}^{2}=0,68\right)$ e Cwb $\left(R^{2}=0,62\right)$, e nos estados de Goiás $\left(R^{2}=0,80\right)$, São Paulo $\left(R^{2}=0,76\right)$ e Minas Gerais $\left(R^{2}=0,70\right)$. As distribuições normal (19,3\%), triangular $(2,2 \%)$ e uniforme $(3,5 \%)$ têm menor participação nos melhores ajustes, mas são importantes nas análises para o período seco.

Termos para indexação: função densidade de probabilidade, planejamento agropecuário, risco climático, teste de aderência.

\section{Functional relations between probable and average rainfall in Brazilian regions and climates}

\begin{abstract}
The objective of this work was to determine the statistical parameters of the probability density function (PDF) with the best fit to the values of decennial rainfall observed in several Brazilian localities, and also to determine the relationship between probable rainfall $\left(75 \%\right.$ probability, $\left.\mathrm{P}_{75 \%}\right)$ and average rainfall $(\overline{\mathrm{P}})$ in these localities. Five PDFs were evaluated (normal, triangular, gamma, exponential, and uniform), adjusted to data from 43 municipalities, from eight states, in four Brazilian regions. The localities were evaluated singly or grouped into states or climate types. The Kolmogorov-Smirnov adherence test was used to assess the statistical adjustment of the PDFs to the data series. Gamma and exponential distributions were the ones which more often had the best fit to the series of decennial rainfall (41.2 and 30.8\%, respectively). The most promising functional relationships between $\overline{\mathrm{P}}$ and $\mathrm{P}_{75 \%}$ were obtained at the climates $\mathrm{Cwa}\left(\mathrm{R}^{2}=0.82\right)$, Aw $\left(\mathrm{R}^{2}=0.70\right)$, As $\left(R^{2}=0.68\right)$, and Cwb $\left(R^{2}=0.62\right)$, and at the states of Goiás $\left(R^{2}=0.80\right)$, São Paulo $\left(R^{2}=0.76\right)$, and Minas Gerais $\left(\mathrm{R}^{2}=0.70\right)$. The normal $(19.3 \%)$, triangular $(2.2 \%)$, and uniform $(3.5 \%)$ distributions have the lowest participation in the best fits, but are important in the dry season analyzes.
\end{abstract}

Index terms: probability density function, agriculture planning, climate risk, adherence test.

\section{Introdução}

A quantificação das precipitações pluviais é importante para o correto dimensionamento de obras, tanto na construção civil quanto na engenharia de água e solo (Frizzone et al., 2005). Em geral, no planejamento agropecuário, a determinação da frequência, além da quantidade e da intensidade das precipitações, também tem grande utilidade, pois a produção agrícola comumente é mais influenciada pela distribuição temporal das chuvas do que pelo volume total precipitado (Martins et al., 2010). Diversos autores (Oliveira \& Carvalho, 2003; Sampaio et al., 2006; Soccol et al., 2010) recomendam que se utilize a precipitação provável no dimensionamento de projetos agrícolas, uma vez que o uso de valores médios pode resultar em super ou subdimensionamentos. 
O padrão pluvial de uma região pode ser descrito, com razoável grau de precisão, a partir de séries históricas consistentes. Assim, pode-se estimar a precipitação provável $\left(\mathrm{P}_{\mathrm{p} \%}\right)$ de uma determinada localidade a partir de séries pluviais quando a função densidade de probabilidade (FDP) e os seus parâmetros se ajustarem aos dados de precipitação observados em um determinado período (Frizzone et al., 2005). A precipitação provável refere-se à lâmina mínima de chuva esperada em dado período do ano, para um determinado nível de probabilidade (Frizzone et al., 2005; Ávila et al., 2009).

Quando as análises envolverem mais de uma FDP, a distribuição para um dado período é selecionada de acordo com o melhor ajuste às séries históricas de precipitação, com a facilidade de estimação de seus parâmetros e com a sua flexibilidade computacional. Os parâmetros da FDP com o melhor ajuste aos dados de precipitação são muito úteis para o planejamento de atividades no meio rural e urbano (Cargnelutti Filho et al., 2004). A FDP gama é normalmente ajustada ao total de chuva em períodos mensais ou menores (Lima et al., 2008). Porém, Gomes (2005) e Gherardi et al. (2008) verificaram que outros tipos de FDP são necessários, principalmente quando a série histórica de dados diários de chuva (grande ou pequena) apresenta poucos registros com precipitação superior a $1 \mathrm{~mm}$ no período (pêntada, decêndio, quinzena ou mês), como ocorre em períodos secos e em veranicos.

Para que haja minimização dos riscos, Jensen (1974) recomenda que não se trabalhe com probabilidades de ocorrência de precipitação menores que 80 e $90 \%$, enquanto Doorenbos \& Pruitt (1977) sugerem probabilidades entre 75 e $80 \%$. Segundo Frizzone et al. (2005), a probabilidade de $75 \%$ é considerada adequada para fins agropecuários. Vale ressaltar, no entanto, que, uma vez determinados os parâmetros e a FDP de melhor ajuste, a precipitação provável pode ser facilmente obtida para diferentes níveis de probabilidade.

A literatura é rica em estudos de estimativa de precipitações prováveis em diferentes localidades (Castro Neto \& Silveira, 1983; Araújo et al., 2001; Oliveira \& Carvalho, 2003; Frizzone et al., 2005; Gomes, 2005; Lyra et al., 2006; Sampaio et al., 2006; Fietz et al., 2008; Ávila et al., 2009; Martins et al., 2010; Soccol et al., 2010; Dallacort et al., 2011). Contudo, ainda são escassos os trabalhos que visam a obtenção de relações funcionais entre medidas de precipitação e seus valores prováveis, obtidos por meio de FDPs para diferentes localidades, regiões e climas brasileiros. As pesquisas na área têm se limitado a destacar as diferenças entre os valores de precipitação provável e média, possivelmente em razão da dificuldade de obtenção de séries longas e representativas, necessárias para o estabelecimento de relações funcionais entre essas precipitações.

O objetivo deste trabalho foi determinar os parâmetros estatísticos da FDP, com melhor ajuste aos valores decendiais de precipitação pluvial, observados em diversas localidades brasileiras, e também determinar a relação entre precipitação provável $(75 \%$ de probabilidade, $\left.\mathrm{P}_{75 \%}\right)$ e precipitação média $(\overline{\mathrm{P}})$ nestas localidades.

\section{Material e Métodos}

Foram avaliadas 43 localidades brasileiras, situadas entre $5,83^{\circ}$ e $29,83^{\circ}$ de latitude Sul e entre $35,88^{\circ}$ e $57,23^{\circ}$ de longitude Oeste, com altitude entre 8 e $1.628 \mathrm{~m}$ (Tabela 1). Os dados de precipitação foram organizados em 37 decêndios por ano. O trigésimo sétimo "decêndio" de cada ano foi composto pelos cinco ou seis (ano bissexto) últimos dias do ano. As séries de dados de precipitação tiveram origem do banco de dados da Agência Nacional de Águas, com exceção das séries relativas às cidades do Estado do Paraná, que foram obtidas do Instituto Meteorológico do Paraná, e das séries referentes às cidades de Piracicaba e São Carlos, obtidas da Escola Superior de Agricultura Luiz de Queiroz e da Embrapa Pecuária Sudeste, respectivamente.

Os cálculos e os procedimentos necessários à condução do presente trabalho foram organizados e realizados com o auxílio de planilha eletrônica, com rotinas desenvolvidas especialmente para essa finalidade, de acordo com as recomendações de Assis (1996), Frizzone et al. (2005), Gherardi et al. (2008) e Bussab \& Morettin (2010). As rotinas são descritas a seguir. Os dados diários de precipitação foram tabulados e agrupados em períodos de dez dias (decêndios), tendo-se separado os decêndios sem ocorrência de precipitação $\left(\mathrm{P}_{\mathrm{o}}\right)$ dos com precipitação diferente de zero. Para evitar inconsistências na estimativa dos parâmetros das FDPs utilizadas, os decêndios com precipitação inferior a $1 \mathrm{~mm}$ foram considerados 
Tabela 1. Características das localidades avaliadas.

\begin{tabular}{|c|c|c|c|c|c|c|}
\hline Cidade & $\begin{array}{c}\text { Série } \\
\text { (anos) }\end{array}$ & Estação & \multicolumn{2}{|c|}{ Latitude } & \multirow[t]{2}{*}{$\begin{array}{c}\text { Altitude } \\
(\mathrm{m})\end{array}$} & \multirow[t]{2}{*}{$\begin{array}{c}\text { Classificação } \\
\text { climática }\end{array}$} \\
\hline & & & $\mathrm{Bah}$ & & & \\
\hline Eunápolis & 18 & Mundo Novo & $-16,23$ & $-39,58$ & 189 & Aw \\
\hline Ilhéus & 20 & Itajuípe (Pirangi) & $-14,67$ & $-39,35$ & 52 & Af \\
\hline Jaguaquara & 18 & Santa Inês & $-13,28$ & $-39,82$ & 667 & Aw \\
\hline Jequié & 18 & Jequié & $-13,87$ & $-40,08$ & 215 & Aw \\
\hline Milagres & 15 & Iaçu (Paraguaçu) & $-12,77$ & $-40,20$ & 419 & Bsh \\
\hline Salvador & 15 & Barra do Gil & $-12,98$ & $-38,63$ & 8 & As \\
\hline \multirow[t]{2}{*}{$\underline{\text { Vitória da Conquista }}$} & 16 & Vitória da Conquista & $-14,87$ & $-40,83$ & 923 & $\mathrm{Aw}$ \\
\hline & \multicolumn{6}{|c|}{ Goiás } \\
\hline Anápolis & 23 & Ouro Verde de Goiás & $-16,22$ & $-49,14$ & 1.017 & Cwa \\
\hline Britânia & 23 & Britânia & $-15,24$ & $-51,16$ & 263 & $\mathrm{Am}$ \\
\hline Ceres & 21 & Ceres & $-15,31$ & $-49,60$ & 571 & Cwa \\
\hline Flores de Goiás & 21 & Flores de Goiás & $-14,44$ & $-47,04$ & 440 & Cwa \\
\hline Itumbiara & 15 & Itumbiara & $-18,42$ & $-49,22$ & 448 & Aw \\
\hline \multirow[t]{2}{*}{$\underline{\text { Porangatu }}$} & 25 & Porangatu & $-13,44$ & $-49,14$ & 396 & Cwa \\
\hline & \multicolumn{6}{|c|}{ Maranhão } \\
\hline \multirow[t]{2}{*}{$\underline{B a l s a s}$} & 20 & Balsas & $-7,52$ & $-46,02$ & 247 & Aw \\
\hline & \multicolumn{6}{|c|}{ Minas Gerais } \\
\hline Itajubá & 18 & São João do Itajubá & $-22,38$ & $-45,45$ & 856 & $\mathrm{Cwb}$ \\
\hline Leopoldina & 18 & Usina Maurício & $-21,47$ & $-42,80$ & 212 & Aw \\
\hline Machado & 18 & Machado 83683 & $-21,67$ & $-45,92$ & 820 & $\mathrm{Cwb}$ \\
\hline Montes Claros & 18 & São João da Vereda & $-16,73$ & $-44,10$ & 648 & $\mathrm{Cwb}$ \\
\hline Nanuque & 18 & Nanuque - Montante & $-17,82$ & $-40,35$ & 103 & $\mathrm{Cwb}$ \\
\hline \multirow[t]{2}{*}{$\underline{\text { Paracatu }}$} & 15 & Paracatu & $-17,22$ & $-46,87$ & 687 & Aw \\
\hline & \multicolumn{6}{|c|}{ Mato Grosso } \\
\hline Cuiabá & 20 & Nossa Senhora do Guia & $-15,37$ & $-56,22$ & 176 & Aw \\
\hline \multirow[t]{2}{*}{ Porto dos Gaúchos } & 15 & Porto dos Gaúchos & $-11,65$ & $-57,23$ & 259 & $\mathrm{Am}$ \\
\hline & \multicolumn{6}{|c|}{ Pernambuco } \\
\hline Afrânio & 15 & Cachoeira do Roberto & $-8,63$ & $-41,15$ & 522 & Bsh \\
\hline Araripina & 15 & Nascente (Olho d'água) & $-7,83$ & $-40,45$ & 622 & Bsh \\
\hline Arcoverde & 15 & Brejo de São José & $-8,52$ & $-37,20$ & 663 & Bsh \\
\hline Barreiros & 15 & Gameleira & $-8,58$ & $-35,38$ & 22 & As \\
\hline \multirow[t]{2}{*}{ Caruaru } & 18 & Caruaru & $-8,28$ & $-35,97$ & 554 & Bsh \\
\hline & \multicolumn{6}{|c|}{ Piauí } \\
\hline$\underline{\text { Santa Filomena }}$ & 15 & Alto Parnaíba & $-9,13$ & $-45,93$ & 277 & Bsh \\
\hline & & & Para & & & \\
\hline Antonina & 26 & Simepar & $-25,01$ & $-48,72$ & 20 & $\mathrm{Cfa}$ \\
\hline Campo Mourão & 5 & Simepar & $-24,01$ & $-52,37$ & 585 & $\mathrm{Cfa}$ \\
\hline Curitiba & 9 & Simepar & $-25,25$ & $-49,16$ & 934 & $\mathrm{Cfb}$ \\
\hline Foz do Iguaçu & 8 & Simepar & $-25,33$ & $-54,34$ & 164 & $\mathrm{Cfa}$ \\
\hline Guaíra & 5 & Simepar & $-24,05$ & $-54,15$ & 220 & $\mathrm{Cfa}$ \\
\hline Guarapuava & 31 & Simepar & $-25,39$ & $-51,45$ & 1.098 & $\mathrm{Cfb}$ \\
\hline Lapa & 18 & Simepar & $-25,77$ & $-49,71$ & 908 & $\mathrm{Cfb}$ \\
\hline Londrina & 27 & Simepar & $-23,23$ & $-51,11$ & 585 & $\mathrm{Cfa}$ \\
\hline Maringá & 5 & Simepar & $-23,55$ & $-51,57$ & 596 & $\mathrm{Cfa}$ \\
\hline Ponta Grossa & 50 & Simepar & $-25,02$ & $-50,02$ & 969 & $\mathrm{Cfb}$ \\
\hline & & & Rio Grande & & & \\
\hline$\underline{\text { Natal }}$ & 17 & Fazenda Potengi & $-5,83$ & $-35,53$ & 30 & As \\
\hline & & & Rio Grand & & & \\
\hline$\underline{\text { São Martinho da Serra }}$ & 15 & Restinga Seca & $-29,83$ & $-53,90$ & 453 & $\mathrm{Cfa}$ \\
\hline & & & São P & & & \\
\hline Campos do Jordão & 15 & Vila Capivari & $-22,72$ & $-45,57$ & 1.628 & $\mathrm{Cwb}$ \\
\hline Piracicaba & 50 & Esalq/USP & $-22,71$ & $-47,63$ & 547 & Cwa \\
\hline São Carlos & 15 & Embrapa & $-21,96$ & $-47,84$ & 860 & Cwa \\
\hline
\end{tabular}


iguais a zero (Assis, 1996). Foram estabelecidas 1.591 distribuições de frequência com os dados das séries de precipitação observadas (43 localidades e 37 decêndios) (Bussab \& Morettin, 2010).

Calcularam-se, então, os parâmetros estatísticos das cinco FDPs testadas (gama, normal, exponencial, triangular e uniforme) com as séries de valores de precipitação decendial observadas (1.591 análises para obter os parâmetros de cada FDP). Os parâmetros determinados foram: alfa e beta, para a distribuição gama; média e desvio-padrão, para a normal; maior valor, menor valor e moda, para a triangular; média, para a exponencial; e maior e menor valor, para a uniforme (Assis, 1996; Frizzone et al., 2005; Bussab \& Morettin, 2010). A aderência dos valores decendiais de precipitação estimados às cinco FDPs foi verificada com o teste de Kolmogorov-Smirnov, a 5\% de probabilidade (7.955 testes) (Assis, 1996; Bussab \& Morettin, 2010).

Após a escolha da FDP que melhor se ajustou aos valores decendiais de precipitação observada, determinou-se a precipitação decendial provável, a $75 \%$ de probabilidade $\left(\mathrm{P}_{75 \%}\right)$, para as 43 localidades estudadas, ao longo dos 37 decêndios dos anos. A $\mathrm{P}_{75 \%}$ refere-se ao valor de precipitação que tenha $75 \%$ de probabilidade de ser igualado ou superado, a cada decêndio: $\mathrm{P}_{75 \%}=\mathrm{P}\left(\mathrm{P}_{75 \%} \in \mathrm{P} \mid \mathrm{p}_{\mathrm{i}} \geq \mathrm{P}_{75 \%}\right)=75 \%$. Como algumas das FDPs analisadas não admitem valores nulos, adotou-se o conceito de distribuição mista (Assis, 1996): $\mathrm{F}(\mathrm{p})=\mathrm{P}_{\mathrm{o}}+\left(1-\mathrm{P}_{\mathrm{o}}\right) \times \mathrm{D}(\mathrm{p})$, em que $\mathrm{F}(\mathrm{p})$ é a função cumulativa de probabilidade da distribuição mista $(\%) ; \mathrm{P}_{\mathrm{o}}$ é a probabilidade de ocorrência de decêndios sem precipitação (\%) ou com valores menores que $1 \mathrm{~mm}$; e $\mathrm{D}(\mathrm{p})$ é a probabilidade estimada com a distribuição cumulativa teórica de melhor ajuste, cujos parâmetros foram determinados na ausência de decêndios sem precipitação (\%).

As relações funcionais entre os valores decendiais de $\mathrm{P}_{75 \%}$ e $\mathrm{P}$ (média, maior valor, menor valor, moda e ausência de precipitação) foram testadas a partir de análises de regressão (linear, potencial, exponencial, logarítmica e polinomial), tendo-se considerado as 43 localidades, analisadas isoladamente ou agrupadas em estados ou tipos climáticos. Para a validação das relações funcionais estudadas, os valores de $\mathrm{P}_{75 \%}$ estimados com as equações foram relacionados com os respectivos valores decendiais de $\mathrm{P}_{75 \%}$ obtidos por outros autores $\left(\mathrm{P}_{75 \%}{ }^{* *}\right)$, para diferentes locais e tipos climáticos - Aw (Fietz et al., 2008) e Cwb (Castro Neto \& Silveira, 1983). Foram realizadas duas análises de validação. Na primeira, comparou-se a $\mathrm{P}_{75 \%}$ ** estimada por Fietz et al. (2008), em Cuiabá, MT, no clima Aw, com a $\mathrm{P}_{75 \%}$ calculada pelo uso dos dados de $\overline{\mathrm{P}}$ decendial desses autores na equação de regressão linear obtida no presente trabalho entre os valores de $\overline{\mathrm{P}}$ e $\mathrm{P}_{75 \%}$, para esse tipo climático. Na segunda análise, comparou-se a $\mathrm{P}_{75 \%}$ ** estimada por Castro Neto \& Silveira (1983), em Lavras, MG, no clima Cwb, com a calculada pelo uso dos dados de $\overline{\mathrm{P}}$ decendial desses autores na equação de regressão linear obtida no presente trabalho entre os valores de $\overline{\mathrm{P}}$ e $\mathrm{P}_{75 \%}$, para esse tipo climático.

\section{Resultados e Discussão}

Vinte e cinco localidades tiveram série média de precipitação decendial menor ou igual à média das 43 localidades analisadas, em 13,9 anos (Tabela 2). $\mathrm{O}$ desvio-padrão das séries de dados de precipitação decendial indicou que, nos períodos mais secos, o ajuste das FDPs testadas aos dados observados foi limitado, em algumas regiões. No entanto, isso não causou maior imprecisão na determinação de $\mathrm{P}_{75 \%}$, já que o valor provável provém de uma distribuição mista, que também considera a probabilidade de ocorrência de decêndios sem precipitação $\left(\mathrm{P}_{\mathrm{o}}\right)$.

De acordo com a frequência em que cada FDP apresentou o melhor ajuste aos dados (Tabela 2), nas 1.591 análises (43 localidades x 37 decêndios) realizadas, verificou-se que as distribuições gama e exponencial ajustaram-se melhor às séries históricas de precipitação agrupadas em decêndio, com frequências de melhor ajuste de 41,2 e $30,8 \%$, respectivamente. A distribuição gama destacou-se principalmente nas localidades com maior série histórica, onde atingiu frequência de melhor ajuste maior que $50 \%$. Para as distribuições normal $(19,3 \%)$, triangular $(2,2 \%)$ e uniforme $(3,5 \%)$, as frequências de melhor ajuste foram menores.

Os parâmetros da FDP que melhor se ajustou aos valores de precipitação decendial têm uma série de aplicações voltadas ao planejamento da agricultura. Os parâmetros obtidos podem servir de base para a realização de programas voltados à estimativa da precipitação provável, semelhantes ao desenvolvido por Gomes et al. (2003), que utilizaram os parâmetros mensais alfa e beta da distribuição gama, determinados 
Tabela 2. Frequência (\%) em que as funções densidade de probabilidade (FDP) analisadas apresentaram o melhor ajuste aos dados de precipitação, no total das avaliações.

\begin{tabular}{|c|c|c|c|c|c|c|c|}
\hline \multirow[t]{2}{*}{ Cidade } & \multicolumn{2}{|c|}{ Série $(\text { anos })^{(1)}$} & \multicolumn{5}{|c|}{ FDP } \\
\hline & Média & DP & Normal & Triangular & Gama & Exponencial & Uniforme \\
\hline & & \multicolumn{6}{|c|}{ Bahia } \\
\hline Eunápolis & 15 & 1,3 & 13,5 & 5,4 & 24,3 & 54,1 & 2,7 \\
\hline Ilhéus & 19 & 1,1 & 10,8 & 0,0 & 62,2 & 27,0 & 0,0 \\
\hline Jaguaquara & 11 & 2,4 & 5,4 & 2,7 & 59,5 & 32,4 & 0,0 \\
\hline Jequié & 12 & 2,4 & 21,6 & 0,0 & 45,9 & 29,7 & 2,7 \\
\hline Milagres & 10 & 1,8 & 10,8 & 5,4 & 37,8 & 43,2 & 2,7 \\
\hline Salvador & 13 & 2,2 & 8,1 & 0,0 & 54,1 & 32,4 & 5,4 \\
\hline \multirow[t]{2}{*}{ Vitória da Conquista } & 13 & 1,7 & 5,4 & 0,0 & 32,4 & 62,2 & 0,0 \\
\hline & & \multicolumn{6}{|c|}{ Goiás } \\
\hline Anápolis & 16 & 7,8 & 29,7 & 0,0 & 37,8 & 29,7 & 2,7 \\
\hline Britânia & 15 & 8,2 & 18,9 & 5,4 & 21,6 & 32,4 & 5,4 \\
\hline Ceres & 14 & 7,2 & 24,3 & 0,0 & 48,6 & 18,9 & 5,4 \\
\hline Flores de Goiás & 13 & 7,4 & 18,9 & 0,0 & 45,9 & 29,7 & 0,0 \\
\hline Itumbiara & 10 & 5,2 & 32,4 & 8,1 & 21,6 & 27,0 & 5,4 \\
\hline \multirow[t]{2}{*}{ Porangatu } & 16 & 9,6 & 24,3 & 5,4 & 43,2 & 18,9 & 2,7 \\
\hline & & \multicolumn{6}{|c|}{ Maranhão } \\
\hline \multirow[t]{2}{*}{ Balsas } & 12 & 7,3 & 16,2 & 0,0 & 43,2 & 27,0 & 2,7 \\
\hline & & \multicolumn{6}{|c|}{ Minas Gerais } \\
\hline Itajubá & 14 & 3,8 & 27,0 & 5,4 & 29,7 & 32,4 & 5,4 \\
\hline Leopoldina & 15 & 3,3 & 10,8 & 2,7 & 56,8 & 29,7 & 0,0 \\
\hline Machado & 14 & 4,0 & 18,9 & 0,0 & 45,9 & 35,1 & 0,0 \\
\hline Montes Claros & 10 & 5,8 & 32,4 & 0,0 & 35,1 & 21,6 & 0,0 \\
\hline Nanuque & 14 & 2,2 & 8,1 & 2,7 & 51,4 & 37,8 & 0,0 \\
\hline \multirow[t]{2}{*}{ Paracatu } & 10 & 4,8 & 27,0 & 0,0 & 32,4 & 18,9 & 13,5 \\
\hline & & \multicolumn{6}{|c|}{ Mato Grosso } \\
\hline Cuiabá & 15 & 6,2 & 18,9 & 5,4 & 45,9 & 24,3 & 5,4 \\
\hline \multirow[t]{2}{*}{ Porto dos Gaúchos } & 11 & 5,8 & 27,0 & 10,8 & 32,4 & 8,1 & 10,8 \\
\hline & & \multicolumn{6}{|c|}{ Pernambuco } \\
\hline Afrânio & 5 & 3,7 & 24,3 & 0,0 & 21,6 & 18,9 & 5,4 \\
\hline Araripina & 8 & 3,8 & 24,3 & 0,0 & 35,1 & 27,0 & 5,4 \\
\hline Arcoverde & 8 & 3,3 & 16,2 & 2,7 & 32,4 & 40,5 & 5,4 \\
\hline Barreiros & 12 & 2,3 & 27,0 & 0,0 & 37,8 & 35,1 & 0,0 \\
\hline \multirow[t]{2}{*}{ Caruaru } & 10 & 4,2 & 10,8 & 0,0 & 48,6 & 37,8 & 2,7 \\
\hline & & \multicolumn{6}{|c|}{ Piauí } \\
\hline Santa Filomena & 10 & 5,3 & 37,8 & 0,0 & 29,7 & 13,5 & 13,5 \\
\hline & & & & & & & \\
\hline Antonina & 25 & 1,5 & 29,7 & 2,7 & 40,5 & 24,3 & 2,7 \\
\hline Campo Mourão & 4 & 1,0 & 51,4 & 0,0 & 13,5 & 32,4 & 0,0 \\
\hline Curitiba & 8 & 1,2 & 18,9 & 5,4 & 40,5 & 32,4 & 2,7 \\
\hline Foz do Iguaçu & 7 & 0,9 & 16,2 & 8,1 & 37,8 & 35,1 & 2,7 \\
\hline Guaíra & 4 & 0,7 & 35,1 & 0,0 & 24,3 & 35,1 & 5,4 \\
\hline Guarapuava & 28 & 1,8 & 10,8 & 2,7 & 67,6 & 18,9 & 0,0 \\
\hline Lapa & 16 & 1,9 & 8,1 & 0,0 & 62,2 & 27,0 & 2,7 \\
\hline Londrina & 22 & 3,6 & 16,2 & 0,0 & 51,4 & 32,4 & 0,0 \\
\hline Maringá & 4 & 0,9 & 37,8 & 0,0 & 24,3 & 27,0 & 8,1 \\
\hline Ponta Grossa & 45 & 4,4 & 8,1 & 0,0 & 70,3 & 21,6 & 0,0 \\
\hline & & & & Rio & lo Norte & & \\
\hline Natal & 11 & 4,0 & 2,7 & 0,0 & 37,8 & 54,1 & 5,4 \\
\hline & & & & & do Sul & & \\
\hline São Martinho da Serra & 13 & 1,3 & 10,8 & 0,0 & 37,8 & 48,6 & 2,7 \\
\hline & & & & & & & \\
\hline Campos do Jordão & 14 & 1,7 & 10,8 & 8,1 & 51,4 & 24,3 & 5,4 \\
\hline Piracicaba & 39 & 9,1 & 2,7 & 2,7 & 62,2 & 32,4 & 0,0 \\
\hline São Carlos & 12 & 3,5 & 21,6 & 2,7 & 35,1 & 35,1 & 5,4 \\
\hline Menor participação & - & - & 2,7 & 0,0 & 13,5 & 8,1 & 0,0 \\
\hline Maior participação & - & - & 51,4 & 10,8 & 70,3 & 62,2 & 13,5 \\
\hline Média das participações & - & - & 19,3 & 2,2 & 41,2 & 30,8 & 3,5 \\
\hline
\end{tabular}


para 380 estações climatológicas no território brasileiro. Esses parâmetros também podem ser utilizados para ampliar programas similares e auxiliar no estudo e na simulação dos balanços hídricos decendiais das localidades avaliadas (Frizzone et al., 2005).

A precipitação decendial média das localidades foi de 41,9 $\mathrm{mm}$; porém, houve grande variabilidade entre as localidades estudadas. Em média, os valores de $\mathrm{P}_{\mathrm{o}}$ nas 43 localidades corresponderam a 23,7\% dos decêndios analisados, ao longo dos anos (Tabela 3).

A $\mathrm{P}_{75 \%}$ corresponde à probabilidade de uma determinada precipitação pluvial ocorrer uma vez a cada 1,33 anos, em média. Por meio deste parâmetro, verificou-se que algumas localidades analisadas (Tabela 3) podem enfrentar grandes problemas quanto ao risco de baixa precipitação. Valores de $\overline{\mathrm{P}}_{75 \%}$ inferiores a $2,0 \mathrm{~mm}$ por decêndio sequer devem ser contabilizados para a realização de projetos de irrigação (Oliveira \& Carvalho, 2003; Sampaio et al., 2006; Soccol et al., 2010). O valor médio de $\mathrm{P}_{75 \%}$, nas 43 localidades, foi de $12,1 \mathrm{~mm}$ por decêndio, o que corresponde a $29 \%$ da precipitação média decendial e a uma diferença absoluta entre esses parâmetros de 29,8 mm por decêndio.

De forma geral, as regressões que buscavam identificar algum tipo de associação entre os valores decendiais de $\overline{\mathrm{P}}, \overline{\mathrm{P}}_{75 \%}, \overline{\mathrm{P}}_{0}, \mathrm{P}_{75 \%}, \mathrm{P}_{\mathrm{Ma}}$ e $\mathrm{P}_{\mathrm{o}}$ resultaram em baixos coeficientes de determinação, com exceção da regressão linear entre $\overline{\mathrm{P}}$ e $\overline{\mathrm{P}}_{75 \%}$ (Tabela 4 e Figura 1). A associação observada entre esses valores indica que há possibilidade de determinar a precipitação provável a $75 \%$ a partir da precipitação média, independentemente de as 43 localidades serem analisadas isoladamente ou agrupadas em estados ou tipos climáticos. Esse resultado é relevante pois pode facilitar consideravelmente a obtenção de valores prováveis de precipitação, uma vez que a precipitação média pode ser facilmente calculada a partir de séries pequenas de dados.

Com o aprofundamento das análises, observou-se estreita relação linear entre os valores decendiais de $\overline{\mathrm{P}}$ e $\mathrm{P}_{75 \%}$ em algumas localidades (Tabela 4). Além disso, constatou-se que a relação entre esses parâmetros pode estar relacionada à condição climática e, possivelmente, às características operacionais (manutenção e tamanho da série disponível, entre outras) das estações, nos diferentes estados (Tabela 5).
As baixas relações observadas entre $\overline{\mathrm{P}}$ e $\mathrm{P}_{75 \%}$ para o clima Bsh ou para o Estado de Pernambuco (Tabela 5) deveram-se aos baixos volumes de precipitação registrados nesses casos, em que foram verificados poucos períodos decendiais com $\mathrm{P}_{75 \%}$ maior do que zero (Figura 2 B). Esse resultado, entretanto, contrasta com os obtidos nas localidades com clima Cwa (Figura 2 A). Os resultados observados para o Estado do Paraná (Tabelas 4 e 5) provavelmente foram prejudicados pelo pequeno tamanho das séries de dados utilizadas. Constatou-se que os coeficientes de determinação $\left(R^{2}\right)$ geralmente foram maiores nas localidades com maior série de dados de precipitação (Tabela 4). As quatro localidades com clima Aw no Estado da Bahia tiveram baixos valores de $\mathrm{R}^{2}$ (Tabela 4 ). No entanto, o $\mathrm{R}^{2}$ obtido para todas as localidades com esse tipo climático foi elevado (Tabela 5).

$\mathrm{Na}$ validação das equações de regressão entre $\overline{\mathrm{P}} \mathrm{e}$ $\mathrm{P}_{75 \%}$, observou-se que os valores de $\mathrm{P}_{75 \%}$ estimados com essas equações, a partir dos dados de $\overline{\mathrm{P}}$ decendial utilizados para a validação (Castro Neto \& Silveira, 1983; Fietz et al., 2008), apresentaram estreita correlação com os valores calculados por esses autores. Os valores de $\mathrm{R}^{2}$ obtidos para a regressão entre a $\mathrm{P}_{75 \%}$ ** estimada pelos autores e a $\mathrm{P}_{75 \%}$ obtida com as equações geradas no presente trabalho foram de 0,874 , para os dados de Fietz et al. (2008), no clima Aw, e de 0,804, para os dados de Castro Neto \& Silveira (1983), no clima Cwb (Figura 3). Esses resultados confirmam a possibilidade de utilização de $\overline{\mathrm{P}}$ para estimar a $\mathrm{P}_{75 \%}$. Cabe destacar que os resultados obtidos em ambas as análises de validação poderiam ter sido ainda melhores se a metodologia utilizada por Castro Neto \& Silveira (1983) e Fietz et al. (2008), para fechar os decêndios, tivesse sido a mesma que a usada no presente trabalho.

No entanto, as séries históricas diárias de precipitação utilizadas não foram ideais, em razão de dificuldades relacionadas à sua obtenção. Portanto, existem algumas fontes de incertezas nas análises, tais como: séries históricas diárias de precipitação provenientes de vários órgãos governamentais, os quais não utilizam o mesmo padrão instrumental e de realização das leituras; algum tipo de falha em todas as séries, cujo período teve de ser descartado; oscilação no número de anos das séries analisadas, para as localidades; e séries de precipitação com poucos anos, em algumas localidades. Contudo, apesar dessas incertezas, os resultados obtidos para a estimativa da $\mathrm{P}_{75 \%}$ a partir da precipitação média foram 
Tabela 3. Resumo das 1.591 análises realizadas ( 37 decêndios por ano, nas 43 localidades avaliadas) ${ }^{(1)}$.

\begin{tabular}{|c|c|c|c|c|c|c|c|c|c|c|}
\hline \multirow[t]{2}{*}{ Cidade } & Anos & $\overline{\mathrm{P}}$ & $\mathrm{S}_{\mathrm{P}}$ & $\mathrm{P}_{\mathrm{Ma}}$ & $\overline{\mathrm{P}}_{75}$ & $\mathrm{~S}_{\mathrm{P} 75 \%}$ & $\mathrm{P}_{\mathrm{Ma} 75 \%}$ & $\overline{\mathrm{P}_{0}}$ & $\mathrm{~S}_{\mathrm{Po}}$ & $\mathrm{P}_{\mathrm{oMa}}$ \\
\hline & & -------- & - & $\mathrm{mm}$ por & ndio) & & ------- & ------- & $(\%)---$ & ----- \\
\hline & & & & & & & & & & \\
\hline Eunápolis & 17,9 & 33,7 & 28,7 & 231,2 & 5,2 & 4,0 & 13,5 & 15,0 & 6,9 & 31,6 \\
\hline Ilhéus & 19,8 & 42,4 & 36,6 & 266,2 & 14,6 & 7,4 & 35,2 & 3,8 & 4,7 & 19,0 \\
\hline Jaguaquara & 18,0 & 22,3 & 23,6 & 259,7 & 0,2 & 0,6 & 3,6 & 38,0 & 12,6 & 68,4 \\
\hline Jequié & 17,9 & 24,5 & 23,9 & 292,6 & 0,6 & 1,4 & 5,8 & 31,6 & 13,0 & 57,9 \\
\hline Milagres & 14,9 & 21,9 & 22,8 & 223,0 & 1,1 & 2,0 & 7,2 & 28,8 & 11,1 & 50,0 \\
\hline Salvador & 14,8 & 59,9 & 52,1 & 412,6 & 18,5 & 20,5 & 82,4 & 11,4 & 13,8 & 62,5 \\
\hline \multirow[t]{2}{*}{ Vitória da Conquista } & 16,0 & 24,6 & 23,9 & 290,2 & 3,1 & 4,2 & 22,6 & 18,0 & 9,7 & 47,1 \\
\hline & \multicolumn{10}{|c|}{ Goiás } \\
\hline Anápolis & 22,9 & 51,1 & 36,2 & 304,2 & 20,2 & 22,4 & 82,3 & 27,0 & 32,9 & 87,5 \\
\hline Britânia & 22,8 & 48,8 & 36,3 & 560,0 & 15,0 & 18,8 & 78,4 & 33,0 & 34,6 & 95,8 \\
\hline Ceres & 21,0 & 48,1 & 33,0 & 377,0 & 17,4 & 19,0 & 59,5 & 31,1 & 32,5 & 90,9 \\
\hline Flores de Goiás & 21,0 & 38,3 & 30,1 & 217,2 & 10,0 & 12,2 & 37,9 & 36,2 & 33,8 & 90,9 \\
\hline Itumbiara & 15,0 & 41,7 & 33,0 & 296,4 & 13,8 & 17,8 & 66,3 & 32,6 & 32,7 & 93,8 \\
\hline \multirow[t]{2}{*}{ Porangatu } & 24,9 & 47,0 & 35,4 & 330,8 & 17,1 & 19,7 & 65,5 & 33,3 & 37,0 & 96,2 \\
\hline & \multicolumn{10}{|c|}{ Maranhão } \\
\hline \multirow[t]{2}{*}{$\underline{B a l s a s}$} & 19,7 & 33,6 & 27,4 & 240,0 & 9,2 & 11,8 & 41,7 & 36,1 & 35,8 & 95,2 \\
\hline & \multicolumn{10}{|c|}{ Minas Gerais } \\
\hline Itajubá & 18,0 & 46,2 & 33,2 & 323,1 & 15,7 & 17,6 & 56,3 & 19,1 & 20,1 & 68,4 \\
\hline Leopoldina & 17,9 & 44,0 & 34,9 & 287,3 & 13,7 & 14,9 & 49,2 & 17,4 & 17,2 & 63,2 \\
\hline Machado & 17,9 & 51,4 & 39,8 & 317,8 & 18,1 & 19,4 & 61,8 & 18,7 & 21,3 & 68,4 \\
\hline Montes Claros & 17,8 & 40,1 & 33,9 & 318,8 & 6,7 & 12,6 & 54,8 & 42,7 & 30,7 & 94,7 \\
\hline Nanuque & 17,9 & 31,7 & 27,9 & 210,4 & 5,1 & 7,8 & 32,7 & 21,4 & 11,7 & 47,4 \\
\hline \multirow[t]{2}{*}{$\underline{\text { Paracatu }}$} & 14,9 & 48,1 & 35,4 & 386,6 & 15,0 & 21,8 & 74,8 & 32,8 & 30,5 & 93,8 \\
\hline & \multicolumn{10}{|c|}{ Mato Grosso } \\
\hline Cuiabá & 20,5 & 50,6 & 41,2 & 285,0 & 16,7 & 17,3 & 50,5 & 24,7 & 29,6 & 81,8 \\
\hline \multirow[t]{2}{*}{ Porto dos Gaúchos } & 14,9 & 59,0 & 31,5 & 301,5 & 33,2 & 32,9 & 105,5 & 22,7 & 34,0 & 87,5 \\
\hline & \multicolumn{10}{|c|}{ Pernambuco } \\
\hline Afrânio & 14,9 & 32,3 & 22,8 & 314,4 & 0,3 & 1,5 & 9,4 & 61,0 & 23,5 & 93,8 \\
\hline Araripina & 15,0 & 25,0 & 22,3 & 188,8 & 1,6 & 4,9 & 27,6 & 45,5 & 24,0 & 93,8 \\
\hline Arcoverde & 15,0 & 26,3 & 20,2 & 184,5 & 4,2 & 16,5 & 100,4 & 40,9 & 20,8 & 87,5 \\
\hline Barreiros & 14,9 & 62,9 & 50,5 & 368,9 & 18,0 & 24,8 & 85,5 & 17,3 & 14,7 & 43,8 \\
\hline \multirow[t]{2}{*}{ Caruaru } & 18,0 & 18,9 & 17,9 & 139,0 & 0,8 & 1,9 & 8,3 & 42,2 & 22,0 & 78,9 \\
\hline & \multicolumn{10}{|c|}{ Piauí } \\
\hline \multirow[t]{2}{*}{ Santa Filomena } & 14,8 & 41,4 & 30,5 & 288,3 & 15,1 & 16,9 & 58,6 & 31,2 & 34,0 & 93,8 \\
\hline & \multicolumn{10}{|c|}{ Paraná } \\
\hline Antonina & 26,1 & 69,5 & 47,0 & 402,6 & 34,3 & 25,1 & 91,8 & 3,1 & 4,7 & 18,5 \\
\hline Campo Mourão & 4,6 & 45,1 & 31,8 & 262,0 & 13,4 & 14,2 & 46,9 & 17,3 & 19,1 & 93,8 \\
\hline Curitiba & 9,3 & 41,4 & 33,3 & 187,0 & 14,6 & 11,0 & 45,3 & 7,8 & 11,7 & 50,0 \\
\hline Foz do Iguaçu & 7,6 & 48,0 & 41,4 & 293,8 & 12,0 & 10,0 & 34,6 & 9,8 & 10,9 & 33,3 \\
\hline Guaíra & 4,5 & 45,0 & 36,2 & 318,4 & 12,7 & 17,1 & 67,6 & 16,2 & 12,8 & 40,0 \\
\hline Guarapuava & 30,5 & 53,7 & 43,8 & 321,2 & 17,6 & 10,4 & 39,7 & 6,5 & 6,4 & 25,0 \\
\hline Lapa & 18,0 & 44,9 & 36,3 & 224,5 & 13,7 & 11,2 & 47,5 & 10,3 & 9,6 & 31,6 \\
\hline Londrina & 26,7 & 47,8 & 42,8 & 308,3 & 10,9 & 11,0 & 33,7 & 16,4 & 13,2 & 46,4 \\
\hline Maringá & 4,7 & 45,0 & 29,5 & 269,0 & 11,8 & 14,2 & 52,2 & 17,0 & 16,4 & 66,7 \\
\hline Ponta Grossa & 50,2 & 44,5 & 36,9 & 286,6 & 12,3 & 9,3 & 30,7 & 10,7 & 8,9 & 28,8 \\
\hline & & & & & io Gra & lo Nor & & & & \\
\hline Natal & 17,0 & 28,1 & 26,8 & 182,0 & 4,0 & 6,4 & 26,7 & 31,7 & 22,1 & 77,8 \\
\hline & & & & & Rio $\mathrm{Gr}$ & do $\mathrm{Sul}$ & & & & \\
\hline São Martinho da Serra & 15,0 & 41,2 & 35,5 & 260,0 & 9,2 & 6,3 & 25,7 & 11,5 & 8,3 & 37,5 \\
\hline & & & & & & & & & & \\
\hline Campos do Jordão & 15,0 & 48,6 & 36,3 & 296,0 & 20,5 & 19,8 & 66,4 & 8,3 & 10,4 & 31,3 \\
\hline Piracicaba & 49,6 & 40,7 & 34,3 & 277,9 & 10,7 & 12,2 & 39,0 & 20,5 & 19,1 & 54,9 \\
\hline São Carlos & 15,3 & 41,5 & 31,3 & 400,0 & 14,0 & 16,1 & 60,6 & 20,5 & 22,0 & 70,6 \\
\hline
\end{tabular}


Tabela 4. Análises de regressão para a relação entre os valores de precipitação média decendial $(\overline{\mathrm{P}})$ e precipitação decendial provável a $75 \%$ de probabilidade $\left(\mathrm{P}_{75 \%}\right)$, para as 43 localidades avaliadas e os 37 decêndios.

\begin{tabular}{|c|c|c|c|c|c|c|}
\hline \multirow[t]{3}{*}{ Cidade } & \multirow[t]{3}{*}{ Anos } & \multirow{2}{*}{ Equação de regressão linear } & \multicolumn{2}{|c|}{ Restrição $^{(1)}$} & \multirow[t]{3}{*}{$\mathrm{R}^{2}$} & \multirow{3}{*}{$\begin{array}{l}\text { Classificação } \\
\text { climática }\end{array}$} \\
\hline & & & $\mathrm{Li}$ & Ls & & \\
\hline & & \multicolumn{3}{|c|}{ 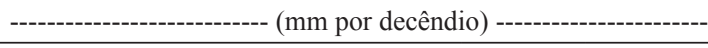 } & & \\
\hline & & & & & & \\
\hline Eunápolis & 17,9 & $\mathrm{P}_{75 \%}=0,109 \overline{\mathrm{P}}+1,520$ & 16,6 & 69,5 & 0,10 & Aw \\
\hline Ilhéus & 19,8 & $\mathrm{P}_{75 \%}=0,346 \overline{\mathrm{P}}-0,070$ & 18,0 & 79,3 & 0,31 & Af \\
\hline Jaguaquara & 18,0 & $\mathrm{P}_{75 \%}=0,000 \overline{\mathrm{P}}+0,159$ & 9,0 & 72,2 & 0,00 & Aw \\
\hline Jequié & 17,9 & $\mathrm{P}_{75 \%}=0,012 \overline{\mathrm{P}}+0,341$ & 6,6 & 67,9 & 0,02 & Aw \\
\hline Milagres & 14,9 & $\mathrm{P}_{75 \%}=-0,001 \overline{\mathrm{P}}+1,133$ & 5,1 & 60,7 & 0,00 & Bsh \\
\hline Salvador & 14,8 & $\mathrm{P}_{75 \%}=0,563 \underline{\overline{\mathrm{P}}}-15,230$ & 27,1 & 137,2 & 0,76 & As \\
\hline \multirow[t]{2}{*}{ Vitória da Conquista } & 16,0 & $P_{75 \%}=0,145 \bar{P}-0,473$ & 5,8 & 75,6 & 0,36 & $\mathrm{Aw}$ \\
\hline & \multicolumn{6}{|c|}{ Goiás } \\
\hline Anápolis & 22,9 & $\mathrm{P}_{75 \%}=0,671 \overline{\mathrm{P}}-14,13$ & 21,1 & 113,2 & 0,89 & Cwa \\
\hline Britânia & 22,8 & $\mathrm{P}_{75 \%}=0,497 \underline{\bar{P}}-9,238$ & 18,6 & 109,3 & 0,71 & $\mathrm{Am}$ \\
\hline Ceres & 21,0 & $\mathrm{P}_{75 \%}=0,564 \underline{\overline{\mathrm{P}}}-9,741$ & 17,3 & 106,4 & 0,87 & Cwa \\
\hline Flores de Goiás & 21,0 & $\mathrm{P}_{75 \%}=0,396 \overline{\mathrm{P}}-5,182$ & 13,1 & 78,8 & 0,67 & Cwa \\
\hline Itumbiara & 15,0 & $\mathrm{P}_{75 \%}=0,562 \overline{\mathrm{P}}-9,661$ & 17,2 & 108,1 & 0,79 & Aw \\
\hline \multirow[t]{2}{*}{ Porangatu } & 24,9 & $\mathrm{P}_{75 \%}=0,533 \overline{\mathrm{P}}-7,988$ & 15,0 & 113,0 & 0,83 & Cwa \\
\hline & \multicolumn{6}{|c|}{ Maranhão } \\
\hline \multirow[t]{2}{*}{$\underline{B a l s a s}$} & 19,7 & $\mathrm{P}_{75 \%}=0,409 \overline{\mathrm{P}}-4,560$ & 11,2 & 74,1 & 0,65 & Aw \\
\hline & \multicolumn{6}{|c|}{ Minas Gerais } \\
\hline Itajubá & 18,0 & $\mathrm{P}_{75 \%}=0,761 \overline{\mathrm{P}}-19,540$ & 25,7 & 101,0 & 0,78 & Cwb \\
\hline Leopoldina & 17,9 & $\mathrm{P}_{75 \%}=0,458 \overline{\mathrm{P}}-6,461$ & 14,2 & 109,3 & 0,86 & Aw \\
\hline Machado & 17,9 & $\mathrm{P}_{75 \%}=0,638 \overline{\mathrm{P}}-14,720$ & 23,1 & 105,0 & 0,83 & Cwb \\
\hline Montes Claros & 17,8 & $\mathrm{P}_{75 \%}=0,293 \overline{\mathrm{P}}-5,118$ & 17,5 & 108,4 & 0,46 & Cwb \\
\hline Nanuque & 17,9 & $\mathrm{P}_{75 \%}=0,319 \overline{\mathrm{P}}-5,000$ & 15,7 & 74,1 & 0,53 & Cwb \\
\hline \multirow[t]{2}{*}{$\underline{\text { Paracatu }}$} & 14,9 & $\mathrm{P}_{75 \%}=0,540 \overline{\mathrm{P}}-10,990$ & 20,4 & 139,4 & 0,75 & Aw \\
\hline & \multicolumn{6}{|c|}{ Mato Grosso } \\
\hline Cuiabá & 20,5 & $\mathrm{P}_{75 \%}=0,596 \overline{\mathrm{P}}-13,470$ & 22,7 & 96,3 & 0,78 & $\mathrm{Aw}$ \\
\hline \multirow[t]{2}{*}{ Porto dos Gaúchos } & 14,9 & $\mathrm{P}_{75 \%}=0,835 \overline{\mathrm{P}}-16,100$ & 19,3 & 131,6 & 0,92 & $\mathrm{Am}$ \\
\hline & \multicolumn{6}{|c|}{ Pernambuco } \\
\hline Afrânio & 14,9 & $\mathrm{P}_{75 \%}=0,017 \overline{\mathrm{P}}-0,301$ & 17,8 & 130,3 & 0,10 & Bsh \\
\hline Araripina & 15,0 & $\mathrm{P}_{75 \%}=0,083 \overline{\mathrm{P}}-0,487$ & 5,9 & 57,7 & 0,09 & Bsh \\
\hline Arcoverde & 15,0 & $\mathrm{P}_{75 \%}=0,007 \overline{\overline{\mathrm{P}}}+4,042$ & 4,6 & 88,7 & 0,00 & Bsh \\
\hline Barreiros & 14,9 & $\mathrm{P}_{75 \%}=0,521 \overline{\mathrm{P}}-14,790$ & 28,4 & 136,9 & 0,60 & As \\
\hline Caruaru & 18,0 & $\mathrm{P}_{75 \%}=0,015 \overline{\mathrm{P}}+0,562$ & 2,2 & 41,7 & 0,01 & Bsh \\
\hline & & & & & & \\
\hline Santa Filomena & 14,8 & $\mathrm{P}_{75 \%}=0,497 \overline{\mathrm{P}}-5,550$ & 11,2 & 96,3 & 0,76 & Bsh \\
\hline & & & & & & \\
\hline Antonina & 26,1 & $\mathrm{P}_{75 \%}=0,728 \overline{\mathrm{P}}-16,380$ & 28,4 & 140,6 & 0,88 & $\mathrm{Cfa}$ \\
\hline Campo Mourão & 4,6 & $\mathrm{P}_{75 \%}=0,248 \overline{\mathrm{P}}+2,210$ & 0,0 & 97,1 & 0,16 & $\mathrm{Cfa}$ \\
\hline Curitiba & 9,3 & $\mathrm{P}_{75 \%}=0,585 \overline{\mathrm{P}}-9,587$ & 21,5 & 73,7 & 0,49 & $\mathrm{Cfb}$ \\
\hline Foz do Iguaçu & 7,6 & $\mathrm{P}_{75 \%}=0,150 \underline{\overline{\mathrm{P}}}+4,802$ & 10,8 & 126,2 & 0,11 & $\mathrm{Cfa}$ \\
\hline Guaíra & 4,5 & $\mathrm{P}_{75 \%}=0,439 \overline{\mathrm{P}}-7,048$ & 16,1 & 126,4 & 0,46 & $\mathrm{Cfa}$ \\
\hline Guarapuava & 30,5 & $\mathrm{P}_{75 \%}=0,526 \overline{\mathrm{P}}-10,750$ & 32,0 & 74,0 & 0,35 & $\mathrm{Cfb}$ \\
\hline Lapa & 18,0 & $\mathrm{P}_{75 \%}=0,709 \overline{\mathrm{P}}-18,120$ & 30,8 & 69,0 & 0,55 & $\mathrm{Cfb}$ \\
\hline Londrina & 26,7 & $\mathrm{P}_{75 \%}=0,598 \overline{\mathrm{P}}-17,620$ & 29,5 & 78,4 & 0,62 & $\mathrm{Cfa}$ \\
\hline Maringá & 4,7 & $\mathrm{P}_{75 \%}=0,165 \underline{\overline{\mathrm{P}}}+4,286$ & 4,1 & 130,4 & 0,09 & $\mathrm{Cfa}$ \\
\hline Ponta Grossa & 50,2 & $\mathrm{P}_{75 \%}=0,723 \overline{\mathrm{P}}-19,860$ & 28,0 & 61,1 & 0,49 & $\mathrm{Cfb}$ \\
\hline & & Rio & orte & & & \\
\hline Natal & 17,0 & $\mathrm{P}_{75 \%}=0,314 \overline{\mathrm{P}}-4,821$ & 15,4 & 60,5 & 0,66 & As \\
\hline & & & & & & \\
\hline São Martinho da Serra & 15,0 & $\mathrm{P}_{75 \%}=0,403 \overline{\mathrm{P}}-7,416$ & 18,5 & 61,3 & 0,47 & $\mathrm{Cfa}$ \\
\hline & & & & & & \\
\hline Campos do Jordão & 15,0 & $\mathrm{P}_{75 \%}=0,644 \overline{\mathrm{P}}-10,770$ & 16,8 & 107,8 & 0,75 & Cwb \\
\hline Piracicaba & 49,6 & $\mathrm{P}_{75 \%}=0,632 \underline{\bar{P}}-15,100$ & 23,9 & 77,9 & 0,89 & Cwa \\
\hline$\underline{\text { São Carlos }}$ & 15,3 & $\mathrm{P}_{75 \%}=0,507 \overline{\mathrm{P}}-7,030$ & 13,9 & 117,2 & 0,73 & Cwa \\
\hline
\end{tabular}

${ }^{(1)}$ Restrição, limites da variável independente (precipitação média, $\overline{\mathrm{P}}$ ) para a qual as equações foram ajustadas; Li, limite inferior da $\overline{\mathrm{P}}$; Ls, limite superior da $\overline{\mathrm{P}}$. 
muito promissores. As estreitas relações obtidas entre $\mathrm{P}_{75 \%}$ e $\overline{\mathrm{P}}$ em algumas localidades, tipos climáticos e estados indicam a possibilidade de fácil obtenção da $\mathrm{P}_{75 \%}$ para localidades sem estação climatológica, ou que apresentam série histórica com poucos anos de dados. Além disso, a estimação de $\mathrm{P}_{75 \%}$ a partir de $\overline{\mathrm{P}}$ pode viabilizar programas de planejamento de atividades agropecuárias mais abrangentes.

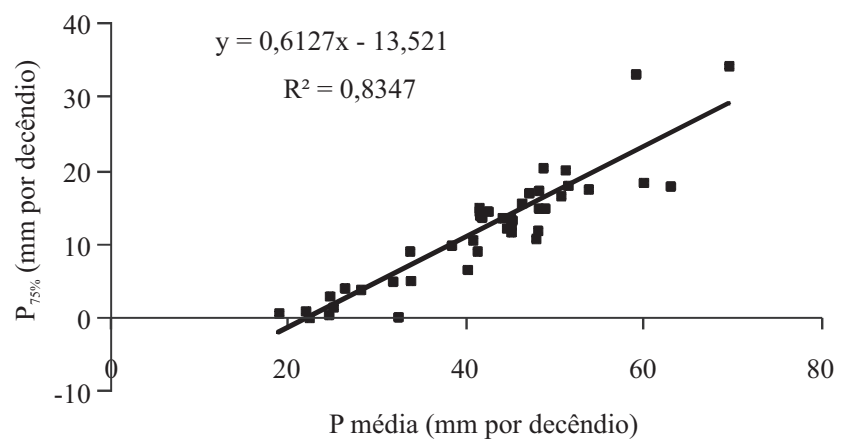

Figura 1. Análise de regressão e coeficiente de determinação $\left(\mathrm{R}^{2}\right)$ entre os valores de precipitação decendial média $(\overline{\mathrm{P}})$ e precipitação provável média a $75 \%\left(\overline{\mathrm{P}}_{75 \%}\right)$. Média obtida para as 43 localidades e os 37 decêndios.

Tabela 5. Análises de regressão obtidas para a relação entre os valores decendiais de $\overline{\mathrm{P}}$ e $\mathrm{P}_{75 \%}$, tendo-se considerado os diferentes tipos climáticos e estados.

\begin{tabular}{|c|c|c|c|c|c|c|}
\hline \multirow[t]{3}{*}{ Parâmetro } & \multirow{2}{*}{$\begin{array}{l}\text { Equação de } \\
\text { regressão linear }\end{array}$} & \multicolumn{2}{|c|}{ Restrição $^{(1)}$} & \multirow[t]{3}{*}{$\mathrm{R}^{2}$} & \multirow[t]{3}{*}{ Lo } & \multirow{3}{*}{$\begin{array}{l}\text { № de } \\
\text { pontos }\end{array}$} \\
\hline & & $\mathrm{Li}$ & Ls & & & \\
\hline & \multicolumn{3}{|c|}{---------(mm por decêndio) ----------- } & & & \\
\hline \multicolumn{7}{|l|}{ Clima } \\
\hline As & $\mathrm{P}_{75 \%}=0,494 \overline{\mathrm{P}}-11,380$ & 23,1 & 137,2 & $0,68^{*}$ & 3 & 111 \\
\hline Aw & $\mathrm{P}_{75 \%}=0,468 \overline{\mathrm{P}}-8,207$ & 17,6 & 139,4 & $0,70^{*}$ & 9 & 333 \\
\hline Bsh & $\mathrm{P}_{75 \%}=0,02 \overline{\mathrm{P}}+1,018$ & 0,0 & 130,3 & 0,00 & 6 & 222 \\
\hline $\mathrm{Cfa}$ & $\mathrm{P}_{75 \%}=0,476 \overline{\mathrm{P}}-8,342$ & 17,6 & 140,6 & $0,48^{*}$ & 7 & 259 \\
\hline $\mathrm{Cfb}$ & $\mathrm{P}_{75 \%}=0,580 \overline{\mathrm{P}}-12,220$ & 21,5 & 74,0 & $0,46^{*}$ & 5 & 185 \\
\hline Cwa & $P_{75 \%}=0,559 \bar{P}-9,965$ & 17,9 & 117,2 & $0,82 *$ & 6 & 222 \\
\hline Cwb & $\mathrm{P}_{75 \%}=0,670 \overline{\mathrm{P}}-10,570$ & 15,8 & 108,4 & $0,62 *$ & 5 & 185 \\
\hline \multicolumn{7}{|l|}{ Estado } \\
\hline Bahia & $\mathrm{P}_{75 \%}=0,379 \overline{\mathrm{P}}-6,232$ & 16,5 & 137,2 & $0,56^{*}$ & 7 & 259 \\
\hline Goiás & $P_{75 \%}=0,547 \bar{P}-9,529$ & 17,5 & 113,2 & $0,80^{*}$ & 6 & 222 \\
\hline Minas Gerais & $\mathrm{P}_{75 \%}=0,509 \overline{\mathrm{P}}-9,855$ & 19,4 & 139,4 & $0,70^{*}$ & 6 & 222 \\
\hline Pernambuco & $\mathrm{P}_{75 \%}=0,304 \overline{\mathrm{P}}-5,088$ & 16,8 & 136,9 & $0,33 *$ & 5 & 185 \\
\hline Paraná & $\mathrm{P}_{75 \%}=0,486 \overline{\mathrm{P}}-8,250$ & 17,0 & 140,6 & $0,46^{*}$ & 10 & 370 \\
\hline São Paulo & $\mathrm{P}_{75 \%}=0,597 \overline{\mathrm{P}}-10,980$ & 18,4 & 117,2 & $0,76^{*}$ & 3 & 111 \\
\hline
\end{tabular}

${ }^{(1)}$ Restrição, limites da variável independente (precipitação média, $\overline{\mathrm{P}}$ ) para a qual as equações foram ajustadas; $\mathrm{Li}$, limite inferior da $\overline{\mathrm{P}}$; Ls, limite superior da $\overline{\mathrm{P}}$. *Significativo pelo teste t, a $\% \%$ de probabilidade.
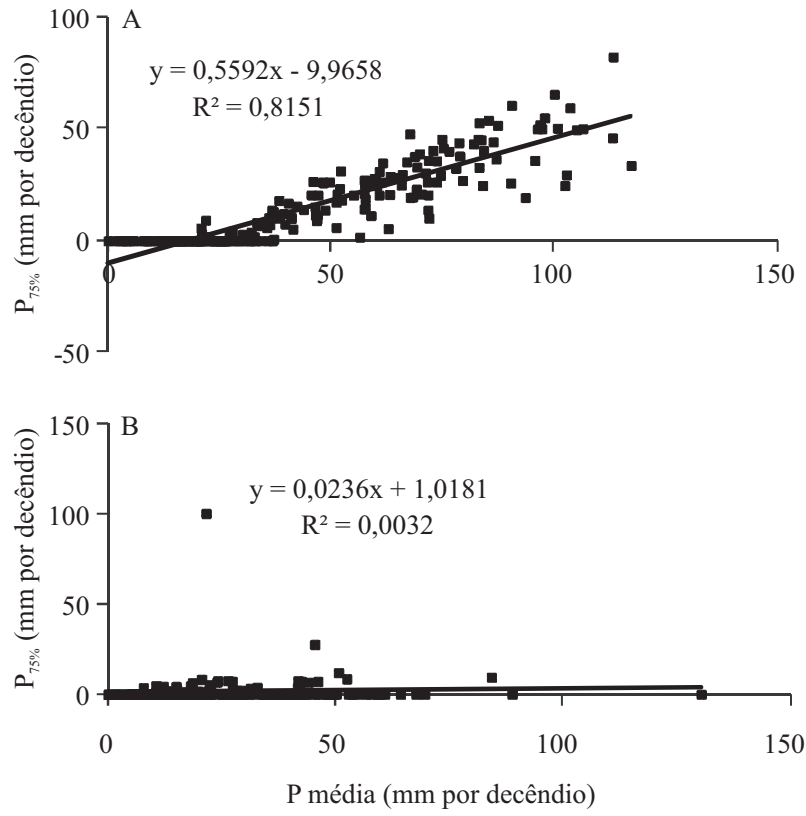

Figura 2. Análises de regressão obtidas para a relação entre os valores decendiais de $\overline{\mathrm{P}}$ e $\mathrm{P}_{75 \%}$, para as localidades com clima Cwa (A) e Bsh (B).
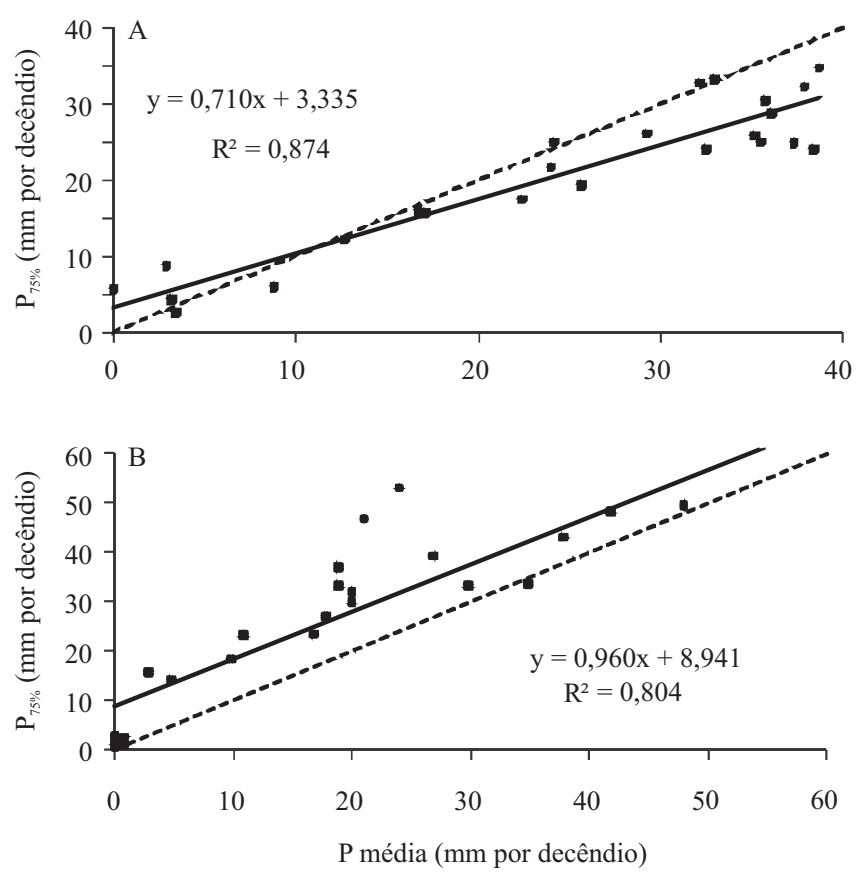

Figura 3. Análises de regressão linear obtidas para a relação entre os valores decendiais da $\mathrm{P}_{75 \%}$ estimada no presente trabalho (climas Aw e Cwb) e das $\mathrm{P}_{75 \%} * *$ calculadas por $\mathrm{A}$, Fietz et al. (2008), em Cuiabá, MT, no clima Aw; e B, por Castro Neto \& Silveira (1983), em Lavras, MG, no clima Cwb. 


\section{Conclusões}

1. As distribuições gama e exponencial se ajustam bem às séries históricas de precipitação decendial estudadas, e a distribuição gama destaca-se principalmente com séries históricas maiores.

2. As distribuições normal, triangular e uniforme apresentam baixas frequências de melhor ajuste aos dados, mas são importantes em análises para períodos secos, que tenham menor número de decêndios com precipitação.

3. As relações funcionais entre $\overline{\mathrm{P}}$ e $\mathrm{P}_{75 \%}$ variam de acordo com os tipos climáticos.

\section{Referências}

ARAÚJO, W.F.; ANDRADE JÚNIOR, A.S. de; MEDEIROS, R.D. de; SAMPAIO, R.A. Precipitação pluviométrica mensal provável em Boa Vista, Estado de Roraima, Brasil. Revista Brasileira de Engenharia Agrícola e Ambiental, v.5, p.563-567, 2001. DOI: 10.1590/S1415-43662001000300032.

ASSIS, F.N.; ARRUDA, H.V.; PEREIRA, A.R. Aplicações de estatística à climatologia: teoria e prática. Pelotas: Ed. da UFPel, 1996. $161 \mathrm{p}$.

ÁVILA, L.F.; MELLO, C.R. de; VIOLA, M.R. Mapeamento da precipitação mínima provável para o sul de Minas Gerais. Revista Brasileira de Engenharia Agrícola e Ambiental, v.13, p.906-915, 2009. DOI: 10.1590/S1415-43662009000700013.

BUSSAB, W. de O.; MORETTIN, P.A. Estatística básica. 6.ed. São Paulo: Saraiva, 2010. 540p.

CARGNELUTTI FILHO, A.; MATZENAUER, R.; TRINDADE, J.K. da. Ajustes de funções de distribuição de probabilidade à radiação solar global no Estado do Rio Grande do Sul. Pesquisa Agropecuária Brasileira, v.39, p.1157-1166, 2004. DOI: 10.1590/ S0100-204X2004001200001.

CASTRO NETO, P.; SILVEIRA, J.V. Precipitação provável para Lavras - MG, baseada na função de distribuição de probabilidade gama III: período de 10 dias. Ciência Prática, v.7, p.58-65, 1983.

DALLACORT, R.; MARTINS, J.A.; INOUE, M.H.; FRETIAS, P.S.L. de; COLETTI, A.J. Distribuição das chuvas no município de Tangará da Serra, médio norte do estado de Mato Grosso, Brasil. Acta Scientiarum. Agronomy, v.33, p.193-200, 2011. DOI: 10.4025/actasciagron.v33i2.5838.

DOORENBOS, J.; PRUITT, W.O. Crop water requirements. Rome: FAO, 1977. 144p. (FAO. Irrigation and drainage paper, 24).
FIETZ, C.R.; COMUNELLO, E.; CREMON, C.; DALLACORT, R. Estimativa da precipitação provável para o Estado de Mato Grosso. Dourados: Embrapa Agropecuária Oeste, 2008. 239p.

FRIZZONE, J.A.; ANDRADE JÚNIOR, A.S.; SOUZA, J.L.M.; ZOCOLER, J.L. Planejamento de irrigação: análise de decisão de investimento. Brasília: Embrapa Informação Tecnológica; Teresina: Embrapa Meio Norte, 2005. 627p.

GHERARDI, B.; DOURADO NETO, D.; SENTELHAS, P.C. Caracterização espacial e temporal da temperatura média do ar, em escala diária e mensal, no Estado de Mato Grosso. Revista Brasileira de Agrometeorologia, v.16, p.33-141, 2008.

GOMES, S. Ajuste e avaliação de um modelo de balanço hídrico decendial e estudo dos limites de sua utilização em algumas localidades no Estado do Paraná. 2005. 103p. Dissertação (Mestrado) - Universidade Federal do Paraná, Curitiba.

GOMES, S.; SOUZA, J.L.M.; MELO, M.C. Probabilidade de ocorrência de chuva mensal. Versão 1.0. Curitiba: UFPR/SCA/ DSEA, 2003.

JENSEN, M.E. Design and operation of farm irrigation systems. New York: American Society of Agricultural Engineers, 1974. 829 p.

LIMA, J.S. de S.; SILVA, S. de A.; OLIVEIRA, R.B. de; CECÍLIO, R.A.; XAVIER, A.C. Variabilidade temporal da precipitação mensal em Alegre - ES. Revista Ciência Agronômica, v.39, p.327-332, 2008

LYRA, G.B.; GARCIA, B.I.L.; PIEDADE, S.M. de S.; SEDIYAMA, G.C.; SENTELHAS, P.C. Regiões homogêneas e funções de distribuição de probabilidade da precipitação pluvial no Estado de Táchira, Venezuela. Pesquisa Agropecuária Brasileira, v.41, p.205-215, 2006. DOI: 10.1590/ S0100-204X2006000200004.

MARTINS, J.A.; DALLACORT, R.; INOUE, A.S.; SANTI, A.; KOLLING, E.M.; COLETTI, A.J. Probabilidade de precipitação para a microrregião de Tangará da Serra, Estado do Mato Grosso. Pesquisa Agropecuária Tropical, v.40, p.291-296, 2010.

OLIVEIRA, L.F.C. de; CARVALHO, D.F. de. Regionalização da lâmina suplementar de irrigação e época de plantio da cultura de feijão, no Estado de Goiás. Revista Brasileira de Engenharia Agrícola e Ambiental, v.7, p.106-110, 2003. DOI: 10.1590/ S1415-43662003000100017.

SAMPAIO, S.C.; LONGO, A.J.; QUEIROZ, M.M.F. de; GOMES, B.M.; VILLAS BOAS, M.A.; SUSZEK, M. Estimativa e distribuição da precipitação mensal provável no Estado do Paraná. Acta Scientiarum. Human and Social Sciences, v.28, p.267-272, 2006. DOI: 10.4025/actascihumansoc.v28i2.169.

SOCCOL, O.J.; CARDOSO, C.O.; MIQUELLUTI, D.J. Análise da precipitação mensal provável para o município de Lages, SC. Revista Brasileira de Engenharia Agrícola e Ambiental, v.14, p.569-574, 2010. DOI: 10.1590/S1415-43662010000600001.

Recebido em 18 de outubro de 2012 e aprovado em 25 de junho de 2013

Pesq. agropec. bras., Brasília, v.48, n.7, p.693-702, jul. 2013

DOI: 10.1590/S0100-204X2013000700001 